\title{
ASKAP Photonic Requirements
}

\author{
Ron Beresford Dec 08 \\ ASKAP Signal and Data Transport IPT \\ Australia Telescope National Facility \\ Commonwealth Scientific Industrial Research Organisation \\ Ron.Beresford@csiro.au
}

\begin{abstract}
The Australian Square Kilometre Array Pathfinder project will be a radio astronomy synthesis array covering the frequency range $700 \mathrm{MHz}-1800 \mathrm{MHz}$ comprised of thirty-six 12m diameter parabolic reflector antennas located in the remote radio quiet desert Midwest region of Western Australia. This paper looks at the architectural requirements for such a system and the role of cost effective photonics solutions required to transport signals within each antenna, intra-array and to the outside world. Photonics solutions include RF over fibre, short-haul and long-haul wavelength division multiplexed (WDM) point to point digital links. A key enabling technology for ASKAP is the formation of multiple beams within the primary field of view (FOV) of the antenna using broadband Focal Plane Array (FPA) feed techniques. At present this is in the experimental phase of development.
\end{abstract}

\section{ASKAP and the SKA}

The Square Kilometre Array (SKA) is a $\$ 1.8$ billion international science project now under consideration: a radio telescope of unprecedented sensitivity able to detect hundreds of times more galaxies, answering fundamental questions in physics and cosmology. The Australian SKA Pathfinder (ASKAP) to be designed and built by the CSIRO in collaboration with overseas teams will provide an important test-bed for SKA technology. The Australian Government has committed over \$100M to ASKAP. Current timelines would see antenna deployment commence at the ASKAP site in the remote Murchison region of Western Australia early 2010. An initial synthesis array of six antennas will comprise the Boolardy Engineering Test Array (BETA). A newly constructed azimuth elevation (Az/El) $12 \mathrm{~m}$ diameter antenna at the Parkes $64 \mathrm{~m}$ observatory will provide the test platform for analyzing FPA performance.

\section{THE ASKAP SYSTEM SPECIFICATION}

Receiver Frequency Range: $700 \mathrm{MHz}-1800 \mathrm{MHz}$

Number of Antennas: $\mathrm{N}_{\mathrm{ANT}}=36$

Number of Beams: 30

$\mathrm{T}_{\text {SYS }}<50 \mathrm{k}$ (after beamforming)

Field of View (FOV): 30 square degrees at $1.4 \mathrm{GHz}$.

Antenna diameter: $12 \mathrm{~m}$

Efficiency: $\varepsilon \sim 60-75 \%$ (after beamforming)

Maximum Baseline: 6km

Collecting Area: Approximately 1\% of SKA

Instantaneous Bandwidth: $300 \mathrm{MHz}$

Spectral Resolution: 19kHz (16000 channels)

The primary purpose of ASKAP is to:

- Demonstrate the low noise, broadband, multiple beam wide FOV focal plane array technique as an applicable SKA technology solution.

- Provide a scientific instrument for furthering radio astronomy research.

- Demonstrate potentially inexpensive SKA solutions.

\section{THE FOCAL PLANE ARRAY}

A focal plane array feed and receiver system together with a $12 \mathrm{~m}$ diameter paraboloid reflector $\mathrm{F} / \mathrm{D}$ ratio $<0.5$ offers a wide field of view to maximize the astronomical survey speed. A dual-polarized, connected "checkerboard" array of $4 \times 5$ elements on a PCB is prototyped (Fig. 2). The final specification is for a 10 x 10 "checkerboard" array. This would require 200 separate receivers and RF/IF signal paths. The output impedance of each array element is approximately $300 \Omega$. Low noise 25-35 Kelvin differential input amplifiers with a $300 \Omega$ input impedance have been designed.

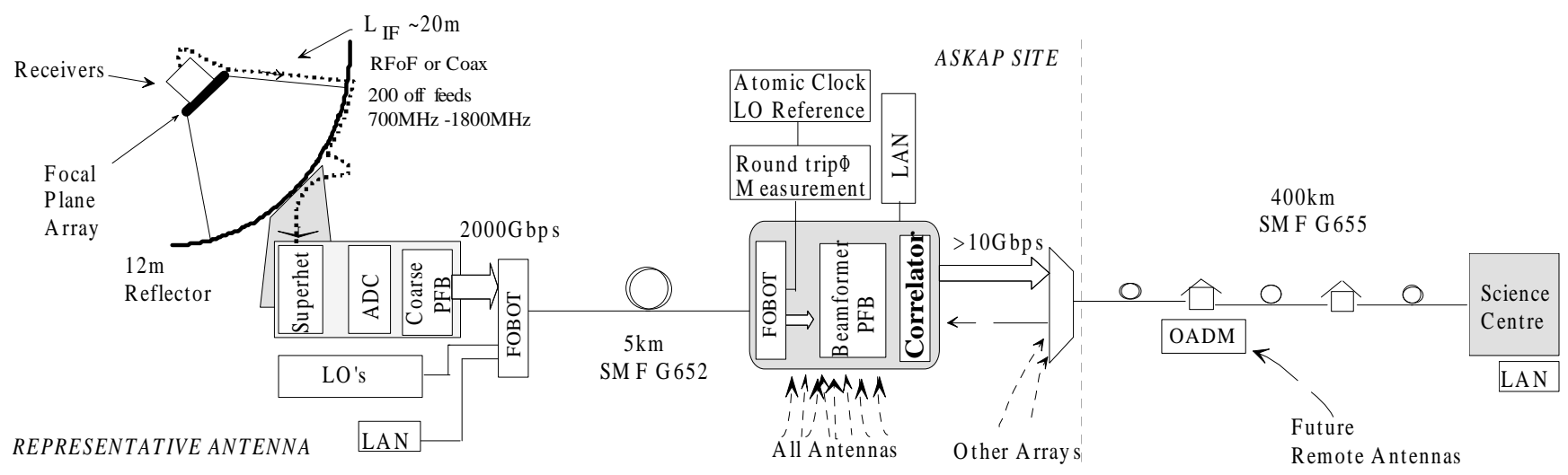

Figure 1. ASKAP System Overview. 


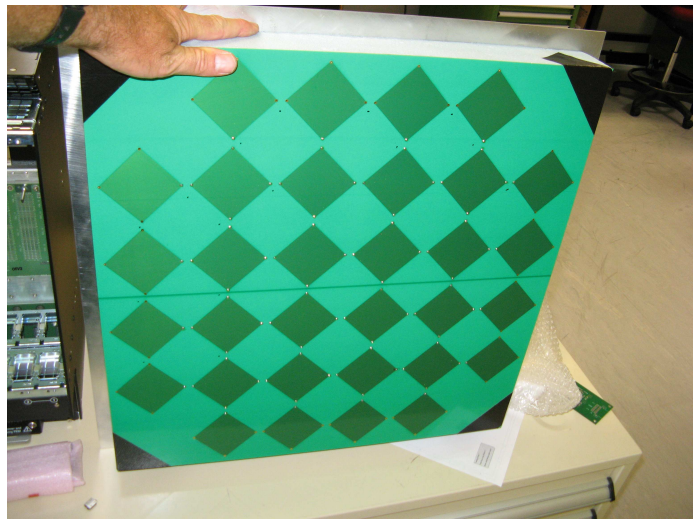

Figure 2. The Prototype 5 x 4 FPA

\section{THE FPA RECEIVERS.}

The final antenna will have 200 receivers. Each receiver has a low noise amplifier (LNA) adjacent to the individual FPA element differential feeds.

The receiver architecture is dependent on the location of the analog to digital converters (ADC). The nearer the ADC proximity to FPA receptors the greater the likelihood of self generated radio frequency interference (RFI) pick-up from the digitizing hardware or electro-optical interface but the signal transmission from the FPA becomes one of data transmission and easy to realize. The further the ADC is from the FPA the more compromising RF signal transmission becomes and it is difficult to preserve the phase stability, amplitude stability and dynamic range.

Currently considered options for receivers are:

- Double conversion super-heterodyne with Local Oscillator (LO) frequencies of $6 \mathrm{GHz}$ and $4 \mathrm{GHz}$ with a 2nd Intermediate Frequency (IF) output of $420 \mathrm{MHz}-720 \mathrm{MHz}$, as shown in (Fig. 3). The IF's are sent to ADC's at the base of the antenna. This is the sub-octave method selected for BETA [3].

- Direct conversion (DC) with quadrature IQ outputs. At present this is in early stages of development in both discrete component assembly and system on a chip (SOC). Some functional blocks for the SOC have been successfully fabricated in 0.18 um CMOS. To preserve the IQ balance the ADC would be required at the mixer possibly introducing RFI at the FPA.

- $\quad$ Single up-conversion with an LO of $6 \mathrm{GHz}$ and 1 st IF bandpass $300 \mathrm{MHz}$ wide at $5 \mathrm{GHz}$ as depicted in (Fig. 3). The IF is down-converted at the base of the antenna and then digitized in the ADC.

- High gain amplification at the LNA and broadband $\mathrm{RF}$ transmission to the receiver downconverter and ADC's at base of antenna. This requires a large gain block at the FPA, typically in the order of $80 \mathrm{~dB}$ presenting intermodulation and stability issues.

- Combined solutions, for example having the downconverters at the focus with digitizers under the dish surface and digital fibre links to the beamformer in the antenna pedestal. RFI could be reduced in this manner.

\section{PHOTONICS SCOPE}

An outline of a proposed ASKAP/BETA signal transmission system is shown (Fig. 1).

1. Transmission of the output from the FPA receivers at the antenna focus to the beamformer ADC input at the pedestal.

2. Short-haul digital transmission of the antenna beamformer digital outputs to the centrally located correlator.

3. Generation of the LO frequencies at antennas from a central master reference.

4. Provision of $1 \mathrm{GE}$ local area network for monitor and control of antennas.

5. Long-haul digital transmission of the correlated ASKAP array output to the science centre for imaging.

\section{Antenna IF Transmission}

Routing the 200 analog signals down the antenna structure and through movable cable tractors on the azimuth and elevation antenna axis (approximately a 20m length) is a significant design issue. The considered options include.

- Coaxial cable for each FPA element, this is bulky for 200 lines of low loss coax. Gain equalization would be required.

- $\quad$ CAT7 cable, this has high attenuation above a few hundred $\mathrm{MHz}$ but is a possibility at low IF frequencies ( less than $1000 \mathrm{MHz}$ ). Gain equalization would be required. Return loss is poor.

- $\quad$ RF over fibre (RFoF), the constraints form factor are eliminated. The noise figure (NF) however is comparable to the high attenuation in coaxial cable for a $20 \mathrm{~m}$ cable run.

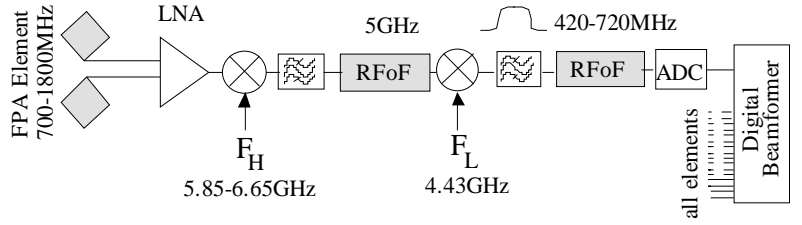

Figure 3. Conversion scheme with RFoF link options. 


\section{RF OVER FIBRE}

Although there are commercial vendors for RF over fiber modules for use in cable television (CATV), antenna remoting and inter-facility communications links, the costs of such modules required for ASKAP would be prohibitive. The ASKAP design would require 7200 short range (typical length $20 \mathrm{~m}$ ) links based on a 36 antenna array of 200 FPA feed elements.

The use of a vertical cavity surface emitting lasers (VCSELs) in the $850 \mathrm{~nm}$ band are considered. These are relatively inexpensive devices (around $\$ 30$ each in quantities of 1000+) designed for digital applications over multimode fibre (MMF). The market push for $10 \mathrm{G}$ Ethernet interfaces has delivered VCSEL devices that can be modulated to $7 \mathrm{GHz}$ or higher.

Bench measurements of directly modulated links using commercial off the shelf VCSEL diodes have been made. The VCSELs were fitted with standard LC style receptacles. Intermediate connections using 12 way multiple parallel optical (MPO) connectors were also performed to assess the suitability of inexpensive multi-way optical quick connects. A variety of prototype printed circuit cards have been developed including standard FR4 material and the low loss Rogers 4003 substrate. Infinicor 50/125um high bandwidth MMF was used in the test configurations. This has a bandwidth distance product of $5 \mathrm{GHzKm}$ and exceeds requirements for a short $20 \mathrm{~m}$ link.

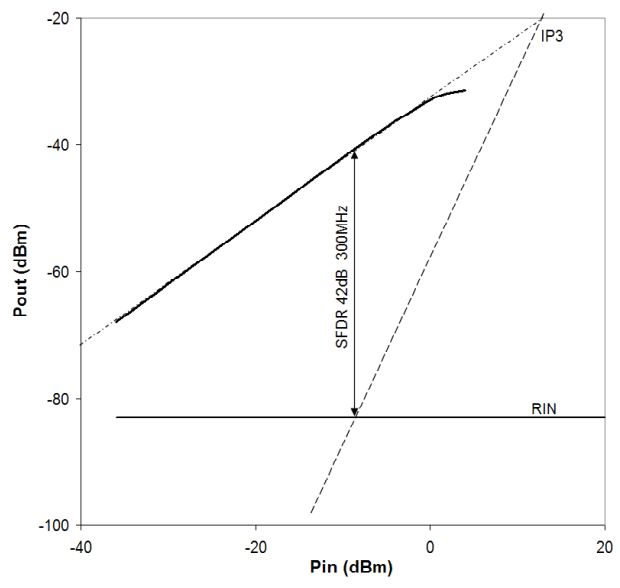

Figure 4. Link dynamic range measurement.

\section{A. Dynamic Range.}

The dynamic range of the short range VCSEL based link is primarily determined by the device linearity $\left(P_{\text {out }}\right.$ versus $I_{f}$ curve) and the relative intensity noise (RIN) of the VCSEL. Using readily available Finisar components with an optical output power of $-3 \mathrm{dBm}$ at a bias of $9 \mathrm{~mA}$ (threshold $5 \mathrm{~mA}$ ) a spurious free dynamic range (SFDR) of $98 \mathrm{dBHz}^{2 / 3}$ was obtained in a $300 \mathrm{MHz}$ bandwidth at a centre frequency of $5 \mathrm{GHz}$. This is an SFDR of $42 \mathrm{~dB}$ in $300 \mathrm{MHz}$ (Fig. 4). A gain $\mathrm{G}=-32 \mathrm{~dB}$ and noise figure $\mathrm{NF}=38 \mathrm{~dB}$ was measured. This could be improved with a higher VCSEL slope efficiency
$\left(\mathrm{P}_{\text {out }}\right.$ versus $\left.\mathrm{I}_{\mathrm{f}}\right)$ characteristic. The link noise floor is (RIN) limited. The measured $\mathrm{RIN}$ is $-140 \mathrm{~dB} / \mathrm{Hz}$ and was $10 \mathrm{~dB}$ better than the quoted manufacturer specification. The $1 \mathrm{~dB}$ compression point was estimated at $\mathrm{P}_{1 \mathrm{~dB}}=-32 \mathrm{dBm}$ and the third order intercept IP3 $=-20 \mathrm{dBm}$. Similar results were obtained with a $300 \mathrm{MHz}$ wide IF at a centre frequency of $600 \mathrm{MHz}$ using VCSELs designed for $3 \mathrm{G}$ data communications purposes. This is acceptable and commensurate with 8 bit ADC's. It is expected that 8 bit quantization in any $300 \mathrm{MHz}$ band for the remote radio quiet ASKAP environment (Fig. 5) is sufficient.

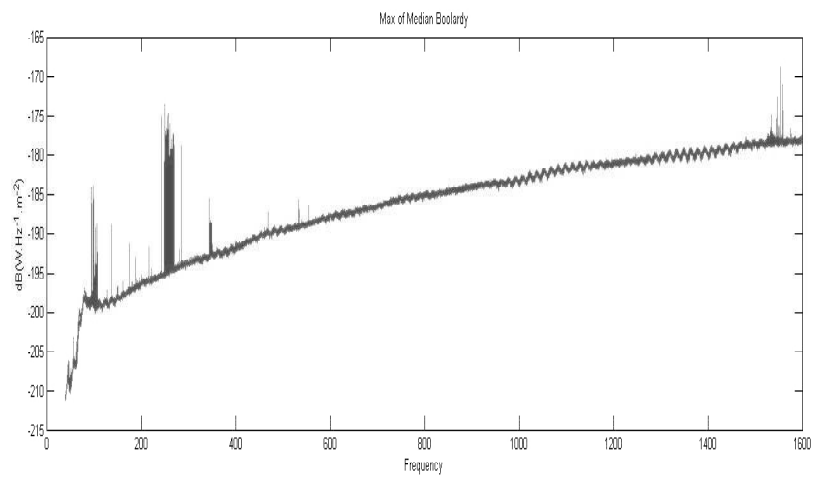

Figure 5. Typical radio quiet spectrum at the ASKAP site.

The VCSEL based MMF links have exhibited sensitivity to fibre movement and bending. Modal noise is generated by the interference between various propagating modes causing speckle pattern (ie amplitude power) variation at the receive photodiode. Using a high power noise diode as a signal source and an integrating spectrometer (Acqiris AC240) as a power meter with consecutive $5 \mathrm{mS}$ integration periods, fluctuations of approximately $0.05 \mathrm{~dB}(1 \%)$ are measured over a bandpass of $400-700 \mathrm{MHz}$ with gentle fiber flexing (Fig. 6).

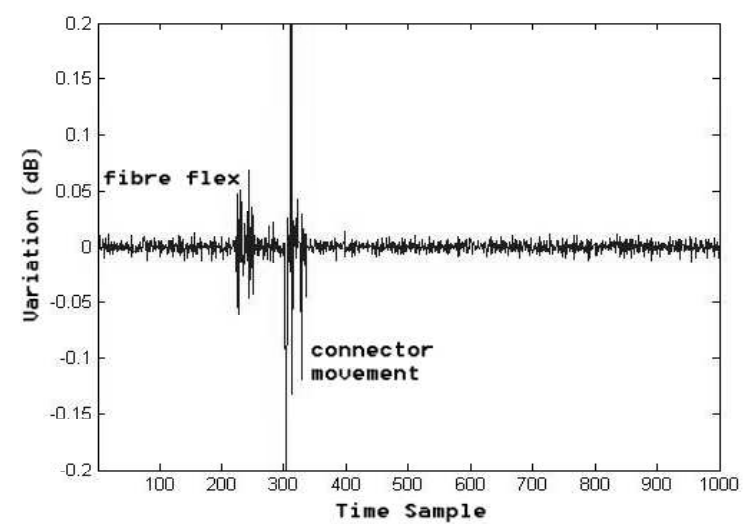

Figure 6. Multimode link amplitude stability.

Averaged over a 60 second period the phase variation should be less than 0.16 degrees and amplitude stability $0.013 \mathrm{~dB}(0.3 \%)$ to meet FPA and interferometer specifications [1]. Larger variations are allowable only if present on timescales much shorter than the integration time. 


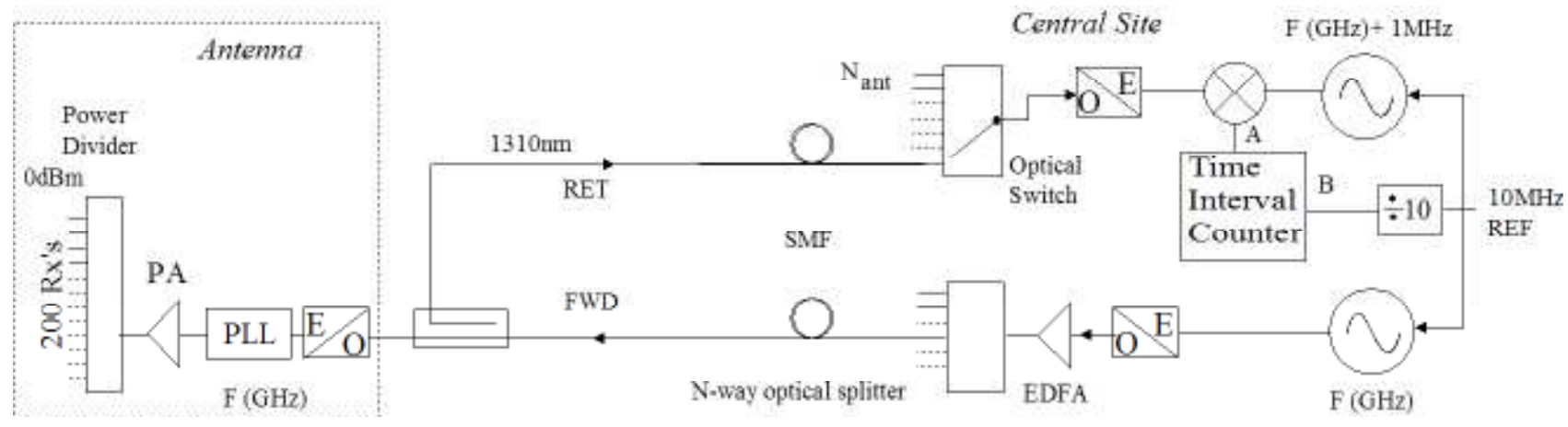

Figure 7. Example round trip LO scheme.

Similar measurements were performed using distributed feedback (DFB) and Fabry-Perot (FP) laser devices at $1310 \mathrm{~nm}$ and $1550 \mathrm{~nm}$. Smaller amplitude variations were measured indicating a bending loss mechanism with fibre flex in singlemode fibre (SMF). Although uncooled FP lasers are available at low cost the use of SMF with 9um core requires more precise connectors and optical alignment, hence is less desirable.

\section{LOCAL OSCILLATOR DISTRIBUTION}

The receiver conversion will require low drift, low phase noise local oscillators. The LO distribution will be on SMF.

The low phase drift is attributed with the SSB noise spectrum of the carrier below a $10 \mathrm{~Hz}$ offset. This is normally associated with temperature coefficient for all components in the local oscillator distribution, namely the temperature coefficient of the fibre and coaxial cable lengths, typically $\sim 10 \mathrm{ppm} /{ }^{\circ} \mathrm{C}$. A round trip phase measurement system can measure the change in electrical path length for the local oscillator signal.

The use of an offset loop (Fig. 7) provides the measurement of phase at the nominally chosen offset frequency (e.g. 1MHz). Experiments with a Stanford Laboratories SR620 precision time interval counter achieves measurements better than 100 picoseconds at $1 \mathrm{MHz}$ (i.e. $0.036^{\circ}$ phase). This is the phase accuracy as it applies at $\mathrm{F}(\mathrm{GHz})$ and exceeds the interferometer specification. The distribution frequencies should be as high as possible to provide maximum measurement sensitivity to the phase changes and to minimize the multiplication of reference local oscillator noise at the antenna.

The use of a phase locked loop (PLL) at each antenna will be required to provide a narrowband clean-up filter to the RIN dominated local oscillator optical receiver output at the antenna. The RIN noise is very wideband at RF/IF frequencies and appears as "LO leakage", perhaps attenuated $30 \mathrm{~dB}$, at the output of mixers used in the receiver conversion process.
It will be difficult to realize an "all optical" local oscillator distribution with RIN that cross correlates between antennas. An all optical system would have had the advantage of reduced RF power levels for the LO's at the FPA and hence less self generated RFI issues.

\section{DATA COMMUNICATIONS}

The short haul data paths will be modular systems based on 10GE small form factor SFP+ packages where possible. Inexpensive SFP+ packages can connect each antenna at $2000 \mathrm{Gbps}$ to the correlator. The total correlator input rate for 45 antennas is $72 \mathrm{Tbps}$. The correlator output rate is a few tens of gigabits per second for long-haul transmission $(\sim 400 \mathrm{~km})$ to the ASKAP science centre.

The long-haul links could be based on carrier class terminal equipment for dense wavelength division multiplexing (DWDM) expansion to terabit per second SKA bandwidths.

\section{ACKNOWLEDGMENT}

I would like to thank Wanxiang Cheng, Paul Roberts and John O'Sullivan for their input to material presented in this paper.

\section{REFERENCES}

[1] John O'Sullivan CSIRO, "Critical ASKAP Analogue Specifications", May 08, unpublished.

[2] Carlsson, Larsson, Alping. "RF Transmission Over Multimode Fibers Using VCSELs - Comparing Standard and High-Bandwidth Multimode Fibers", JLT, vol.22, No7, July 2004, pp.1694-1700.

[3] Jeganathan CSIRO, "ASKAP Double Conversion Receiver and its Integration”, April 08, unpublished.

[4] Paul Roberts CSIRO, "RF Over Fibre link Measurements at 5GHz" Nov 07, unpublished. 